\title{
Structure of amorphous selenium studied by neutron diffraction
}

\author{
Hansen, Flemming Yssing; Knudsen, Torben Steen; Carneiro, K.
}

Published in:

Journal of Chemical Physics

Link to article, DOI:

10.1063/1.430621

Publication date:

1975

Document Version

Publisher's PDF, also known as Version of record

Link back to DTU Orbit

Citation (APA):

Hansen, F. Y., Knudsen, T. S., \& Carneiro, K. (1975). Structure of amorphous selenium studied by neutron diffraction. Journal of Chemical Physics, 62(4), 1556-1565. https://doi.org/10.1063/1.430621

\section{General rights}

Copyright and moral rights for the publications made accessible in the public portal are retained by the authors and/or other copyright owners and it is a condition of accessing publications that users recognise and abide by the legal requirements associated with these rights.

- Users may download and print one copy of any publication from the public portal for the purpose of private study or research.

- You may not further distribute the material or use it for any profit-making activity or commercial gain

- You may freely distribute the URL identifying the publication in the public portal

If you believe that this document breaches copyright please contact us providing details, and we will remove access to the work immediately and investigate your claim. 


\title{
Structure of amorphous selenium studied by neutron diffraction
}

\author{
F. Yssing Hansen and T. Steen Knudsen \\ Fysisk-Kemisk Institut, The Technical University of Denmark, DK 2800 Lyngby, Denmark
}

K. Carneiro*

Danish Atomic Energy Research Establishment, Risø, DK 4000 Roskilde, Denmark

(Received 3 July 1974)

\begin{abstract}
Neutron diffraction measurements on amorphous selenium have been performed at 293 and $80 \mathrm{~K}$. Careful analyses of the instrumental corrections were made to avoid systematic errors in the measured structure factor $S(\kappa)$ in the wave vector region $0 \leq \kappa \leq 12 \AA^{-1}$. As a result of the data treatment, the neutron scattering cross sections of selenium are determined to be $\sigma_{\text {coh }}=8.4 \pm 0.1 \mathrm{~b}$ and $\sigma_{\text {inc }}=0.1 \pm 0.1 \mathrm{~b}$. Using the fact that $S(\kappa)$ for large $\kappa$ 's is determined by the short distances in the sample, a new method for extrapolation of the experimental $S(\kappa)$ until convergence is proposed. This allows an accurate determination of $g(r)$ showing peaks at distances that agree well with the previous' $\mathrm{x}$-ray experiment by Kaplow et al. for distances up to $11 \AA$. However, only partial agreement is obtained when finer details of $g(r)$, e.g., the width of the first peak, are considered. Finally, we give a brief discussion of the different models for the structure of amorphous selenium, taking both diffraction measurements and thermodynamic considerations into account.
\end{abstract}

\section{INTRODUCTION}

In this paper we present the results of neutron diffraction experiments from amorphous selenium at the temperatures $T=293$ and $80 \mathrm{~K}$, and discuss the information gained on the structure of this material. Several diffraction experiments have been published on this material, both by means of neutron diffraction ${ }^{1,2}$ and $x$-ray diffraction, ${ }^{1,3-5}$ but except for the work by Kaplow etal., ${ }^{4}$ it seems to us that the data analysis may be seriously questioned. We consequently examine in detail how insufficiency in the data treatment influences the final result of a diffraction experiment. The agreement between the results of Kaplow et al. and ours indicates that if the structure of amorphous selenium should be further il luminated, experiments should be performed at larger wave vectors than in the two experiments.

The relation between the structure factor $S(\kappa)$ measured by a diffraction experiment and the pair-distribution function $g(r)$ is given by the relations

$$
\begin{aligned}
& 4 \pi r \rho_{0}[g(r)-1]=\frac{2}{\pi} \int_{0}^{\infty} \kappa[S(\kappa)-1] \sin (\kappa r) d \kappa, \\
& \kappa[S(\kappa)-1]=\int_{0}^{\infty} 4 \pi r \rho_{0}[g(r)-1] \sin (\kappa r) d r,
\end{aligned}
$$

for isotropic samples with a random orientation of the characteristic distances. This includes gases, liquids, amorphous solids, and polycrystalline materials.

In (1) and (2), $\rho_{0}$ is the mean density of the sample, $r$ is the distance from a central atom, and $\kappa$ is the wave vector transfer of the neutrons. The structure factor is determined from the intensity of scattered neutrons as a function of wave vector transfer. It may be obtained from the scattering law $S(\kappa, \omega)$ integrated over all frequencies $\omega$, and may give information about the dynamics of the sample, when measured at different temperatures.

It is important to remember that the pair-distribution function in (1) is only an average distribution function, which does not give the actual distribution of atoms around any atom in the sample. If $g_{l} \cdot\left(\mathbf{r}_{l}{ }^{\prime}\right)$ gives the dis tribution of atoms around atom $l^{\prime}$ and $\mathbf{r}_{l^{\prime} l}=\mathbf{r}$ is the distance between atoms $l^{\prime}$ and $l, g(r)$ may be written as

$$
g(\mathbf{r})=\frac{1}{N} \sum_{l=1}^{N} \sum_{l^{*}=1}^{N} g_{l^{\prime}}\left(\mathbf{r}_{l^{\prime} l}\right),
$$

where $N$ is the number of atoms in the sample. The technique applied as well as the instrumental corrections used are similar to what has previously been used for the structure determination of liquid neon, ${ }^{6}$ and in Sec. II we mention the relevant experimental details. Section III contains the data corrections necessary to obtain $S(\kappa)$ and presents the results for the temperatures 80 and $293 \mathrm{~K}$ for $0 \leq \kappa \leq 12 \AA^{-1}$.

It should be emphasized that the primary experimental result is the structure factor, but since the atomic structure is much clearer revealed by $g(r)$, we derive this function in Sec. IV. However, because of the wave vector cutoff in the experiment, there is no unique way of deriving $g(r)$ from Eq. (1), and consequently approximations must be introduced. We propose a solution to this problem in terms of a model, which in a natural way allows an extension of the measured structure factor. Our method differs from that used in Ref. 4. A more satisfactory solution would be to extend the measurements to larger $\kappa$ values, if possible.

In Sec. $V$ we discuss the results and compare to the ones previously reported. We also discuss briefly how the various proposed models fit the experimental data. As mentioned in the beginning of this section the radial distribution function does not yield the atomic structure, but important characteristics are revealed by a diffraction experiment. By further taking alternative information into account, e.g., calorimetric, we feel that a good test on proposed models is available.

\section{EXPERIMENTAL DETAILS}

The neutron diffraction patterns were obtained on the two-axis spectrometers at the DR 3 reactor at Ris $\varnothing$. In order to optimize the data collection with regard to in- 
tensity, instrumental resolution, and wave vector trans fer range, two different wavelengths have been chosen to cover the range from $0.8 \leq \kappa \leq 12.5 \AA^{-1}$, in steps of 0.05 $\AA^{-1}$. We use $\kappa=4 \pi \sin (\theta) / \lambda$, where $2 \theta$ is the total scatter ing angle and $\lambda$ is the wavelength. The two regions overlap each other in order to match the patterns measured with different wavelengths. Typical spectrometer parameters used for the room temperature setup are shown in Table $I$. The second order contaminations of the neutron beams were in both cases negligible. The incident monochromatic neutrons were counted by a ${ }^{3} \mathrm{He}$ monitor, and the number of diffracted neutrons was obtained from a $\mathrm{BF}_{3}$ counter for a preset number of monitor counts at every wave vector transfer.

The samples were provided by Professor R. O. Pohl, Cornell University, and the density was reported to be $4.25 \mathrm{~g} / \mathrm{ml}$, giving a particle density of $\rho_{0}=0.0324$ atoms $\AA^{-3}$. They were cast in cylindrical shape with a diameter of $1.37 \mathrm{~cm}$ and a height of $3.50 \mathrm{~cm}$. At liquid nitrogen temperature, the sample was mounted in a cryostat, the scattering from which was reduced by masking of the beam.

\section{DATA CORRECTIONS}

In Fig. 1 we show the diffraction pattern at room temperature obtained as described in Sec. II, and similar results were found at liquid nitrogen temperature. For comparison we show a diffraction pattern for a polycrystalline sample. After subtraction of background we get $I(\lambda, \kappa)$. To obtain the structure factor $S(\kappa)$ from $I(\lambda, \kappa)$ we use the following expression, given by de Graaf et al. ${ }^{6}$ :

$$
\begin{aligned}
I(\lambda, \kappa)= & A(\lambda) T(\lambda, \kappa)\left\{S(\kappa)+f_{p}(\lambda, \kappa)\right. \\
& \left.+\left(\sigma_{\text {inc }} / \sigma_{\text {coh }}\right)\left[1+f_{p}(\lambda, \kappa)\right]+\sigma_{m}(\lambda) / \sigma_{\mathrm{coh}}\right\},
\end{aligned}
$$

where $A(\lambda)$ is a normalization constant. Discussed below are the transmission factor $T(\lambda, \kappa)$, the multiple scattering correction $\sigma_{m}(\lambda) / \sigma_{\text {coh }}$, the Placzek correction $f_{p}(\lambda, \kappa)$, and the incoherent correction $\sigma_{1 \mathrm{nc}} / \sigma_{\text {coh }}$. Typical values for the corrections are shown in Table II.

\section{A. The transmission factor $T(\lambda, \kappa)$}

Owing to both scattering and "true" absorption of the incident neutrons, we have introduced in Eq. (4) the wavelength - and angular-dependent transmission factor $T(\lambda, \kappa)$ :

$$
T(\lambda, \kappa)=\frac{1}{V} \int_{V} e^{-\mu\left(L_{1}+L_{2}\right)} d V
$$

$T(\lambda, \kappa)$ is given in the usual way in Eq. (5), where $\mu$ is the sum of the inverse of the scattering length and the

TABLE I. Spectrometer parameters used for the scans performed as described in the text.

\begin{tabular}{llll}
\hline $\begin{array}{l}\text { Incident } \\
\text { wavelength } \\
\lambda[\AA]\end{array}$ & $\begin{array}{l}\text { Monochromator } \\
\text { crystal }\end{array}$ & $\begin{array}{l}\text { Wave vector } \\
\text { transfer range } \\
\kappa\left[\AA^{-1}\right]\end{array}$ & $\begin{array}{l}\text { FWHM } \\
\text { resolution } \\
{\left[\AA^{-1}\right]}\end{array}$ \\
\hline 1.705 & Ge $(1,1,1)$ & $0.60-5.50$ & 0.1 \\
0.852 & Be $(0,0,2)$ & $4.50-12.45$ & 0.3 \\
\hline
\end{tabular}

absorption length. The path length of the neutrons before arriving to the volume element $d V$, from which they are "absorbed," and the path length of the scattered neutrons are denoted $L_{1}$ and $L_{2}$, respectively. $V$ is the volume of the sample. Equation (5) has previously ${ }^{7}$ been evaluated for samples of cylindrical shape. In Ref. 7 the "absorption factor" $1 / T(\lambda, \kappa)$ is tabulated.

In the case of selenium, both the scattering and the absorption cross sections are rather high. Furthermore, the size of the sample makes the correction significant. An earlier work ${ }^{1}$ on amorphous selenium with almost the same sample size revealed two structure factors and pair-distribution functions measured with 0.798 and $1.083 \AA$ neutrons, respectively, but the absorption correction was not included in the data treatment. This may explain the observed differences since the correction is rather small (order of $1-2 \%$ ) for the $0.798 \AA$ measurements, while it is greater for the $1.083 \AA$ measure ments (order of $5-10 \%$ ).

\section{B. Multiple scattering correction $\sigma_{m}(\lambda) / \sigma_{\text {coh }}$}

The diffraction data contain counts from single scattered neutrons as well as from multiple scattered neutrons.

To correct for the multiple scattering we have used the results calculated for cylindrical samples by Blech and Averbach. ${ }^{8}$ Their results are exact for an incoherent scatterer, whereas they underestimate the multiple scattering from strongly coherent scatterers. In our case, however, $S(\kappa)$ does not deviate much from the incoherent value of unity, so we expect the incoherent approximation to be satisfactory.

\section{Placzek correction $f_{p}(\lambda, \kappa)$}

To obtain the structure factor one has to perform an integration of the scattering law $S(\kappa, \omega)$ over all energy transfers $\omega$ for constant $\kappa$ according to the equation

$$
S(\kappa)=\int_{-\infty}^{\infty} S(\kappa, \omega) d \omega
$$

By diffraction measurements one performs the integration of the function $\left(k / k_{0}\right) S(\kappa, \omega)$ at constant angle, where $k$ is the wave vector of the outgoing neutrons, and $k_{0}=2 \pi / \lambda$. The Placzek correction, ${ }^{9}$ necessary to correct for this incorrect integration, has been shown to be

$$
f_{p}(\lambda, \kappa)=\left(\frac{k T}{2 E_{0}}-\frac{\kappa^{2}}{k_{0}^{2}}\right) \frac{m}{M}+\left(\frac{1}{2} \frac{\kappa^{2}}{k_{0}^{2}}+\frac{3}{8} \frac{\kappa^{4}}{k_{0}^{4}}\right)\left(\frac{m}{M}\right)^{2}+\ldots,
$$

where $k_{B}$ is Boltzmann's constant, $T$ the temperature, $m$ the mass of neutrons, and $M$ the mass of the atoms in the sample. $E_{0}=\hbar^{2}\left(k_{0}^{2} / 2 m\right)$ is the energy of the incident neutrons. The contribution from the coherent scattering to the intensity as given in Eq. (4) is

$$
\left[S(\kappa)+f_{p}(\lambda, \kappa)\right] \sigma_{\mathrm{coh}} \text {. }
$$

\section{Incoherent scattering correction $\sigma_{\text {inc }} / \sigma_{\text {coh }}$}

The incoherent structure factor is given as

$$
S_{1 \mathrm{ne}}(\kappa)=\int_{-\infty}^{\infty} S_{1 \mathrm{nc}}(\kappa, \omega) d \omega=1 \text {. }
$$




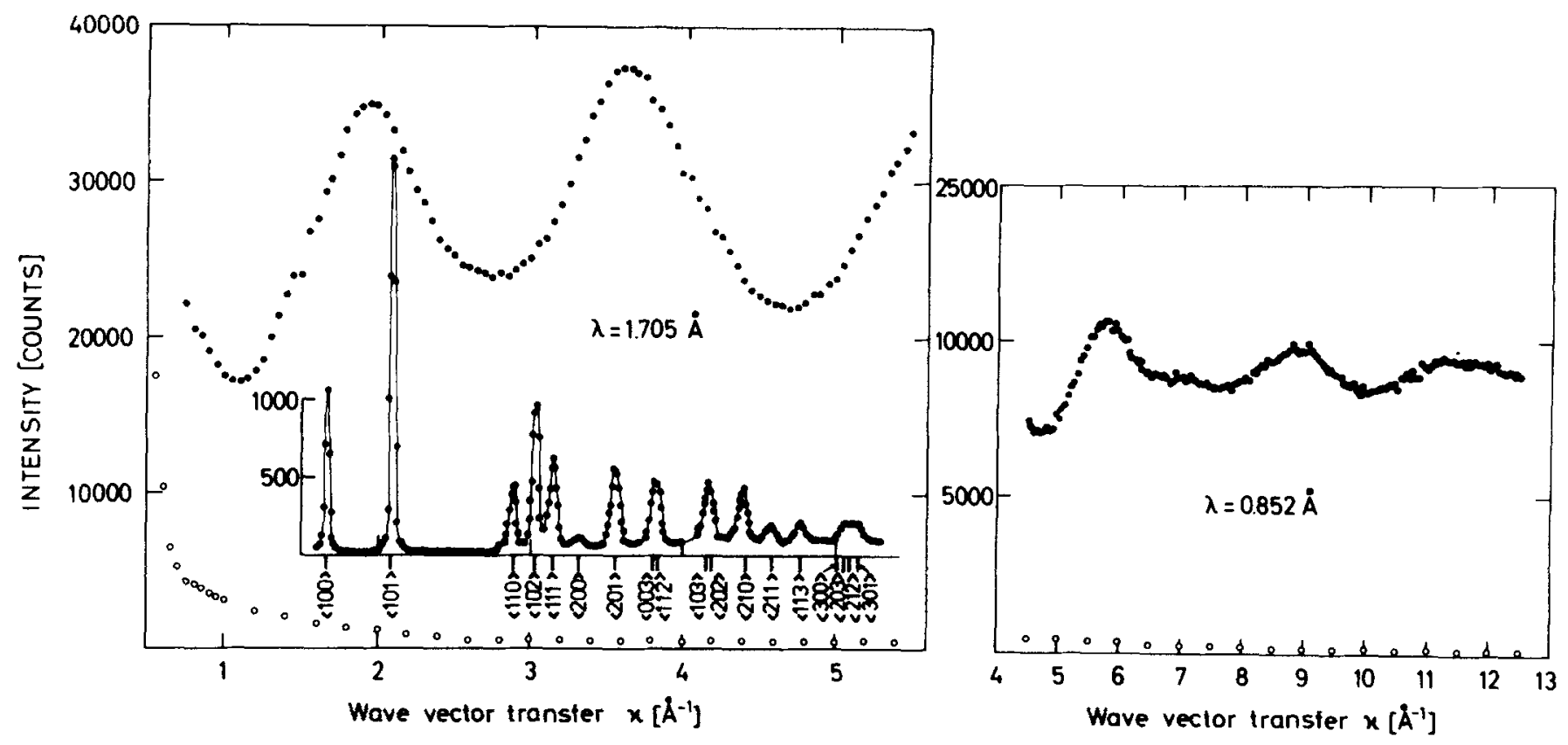

FIG, 1. Diffraction patterns obtained at room temperature from amorphous selenium. Open circles mark background counts. A diffraction pattern for polycrystalline selenium is inserted.

Similar to Eq. (8), the incoherent contribution to the intensity becomes

$$
\left[1+f_{p}(\kappa)\right] \sigma_{\text {inc }} \text {. }
$$

\section{E. Determination of $A(\lambda), \sigma_{i n c}$, and $\sigma_{c o h}$}

To calculate $S(\kappa)$ from $\mathrm{Eq}$. (4) we need the parameters $A(\lambda), \sigma_{1 \mathrm{ac}}$, and $\sigma_{\mathrm{coh}}$. These were determined by making use of the following analytical properties of $S(\kappa)$ and $g(r)$ :

$$
\begin{aligned}
& S(\kappa)-\rho_{0} k T \chi_{T}, \quad \text { for } \kappa \rightarrow 0 \\
& S(\kappa) \rightarrow 1, \text { for } \kappa \rightarrow \infty \\
& g(r)-0, \text { for } r \leq R_{0} .
\end{aligned}
$$

In (10a) $\chi_{T}$ is the isothermal compressibility which in glasses may be set to zero; (10a) therefore allows us to extrapolate $S(\kappa)$ for $0 \leq \kappa \leq 0.8 \AA^{-1}$, where reliable data have not been obtained.

Equation (10b) was used to determine an initial value of $A(0.852 \AA)$. In the overlap region a least square fit was used to determine the corresponding $A(1.705 \AA$ ).

In $(10 \mathrm{c}), R_{0}$ is of the order of the hard-core diameter of selenium. We have used $R_{0}=1.5 \AA$. Unfortunately, the scattering cross section for selenium is rather poorly known. It is reported ${ }^{10}$ that both the coherent and the total scattering cross section is $9 \pm 1 \mathrm{~b}$. The incoherent cross section is not given. The absorption cross section, which is directly proportional to the neutron wavelength, is $7,4 \mathrm{~b}$ for $\lambda=1.08 \AA$ neutrons. ${ }^{10}$ The use of false cross sections in the calculation of the pair-distribution function may result in erroneous distribution functions, so we decided to consider the scattering cross sections as unknown, within the frame of the uncertainties reported. We then optimized our calculation of the pair-distribution function in the following way. Using preliminary values for $\sigma_{\text {con }}$ and $\sigma_{\text {inc }}$ we calculated the Fourier transform of the structure factor according to Eq. (1) in the region $r \leq R_{0}$, where $g(r)$ according to (10c) is zero. The reason for doing so is that normalization errors and errors in the cross section mainly appear for small $r$ values as sharp oscillations, while they die out for greater $r$. As $g(r)-0$ for small $r$ values, we have that $4 \pi r \rho_{0}[g(r)-1] \rightarrow-4 \pi r \rho_{0}$ in this range. Therefore the criterion for the optimal set of the three variables is a least square fit to the line $-4 \pi p_{0} r$. In this way, both from the room temperature data and from the liquid nitrogen temperature data, we found the cross sections to be

$$
\begin{aligned}
& \sigma_{\text {coh }}=8.4 \pm 0.1 \mathrm{~b}, \\
& \sigma_{\text {inc }}=0.1 \pm 0.1 \mathrm{~b} .
\end{aligned}
$$

As the incoherent cross section is very small, it is obvious that the credibility of the value depends very much on the other corrections made, some of those being greater than the incoherent cross section.

A very similar method was used by Kaplow et al. ${ }^{11}$ to determine the $\mathrm{x}$-ray scattering factor of mercury, lead, and tin.

TABLE II. Parameters of importance for the instrumental corrections.

\begin{tabular}{lll}
\hline \hline$\lambda[\AA]$ & 1.705 & 0.852 \\
\hline$T(\lambda, \kappa)$ & $0.610-0.642$ & $0.482-0.508$ \\
$f_{p}(\lambda, \kappa)$ & $5 \times 10^{-3}-(-) 2.2 \times 10^{-2}$ & $(-) 6 \times 10^{-3}-(-) 3.4 \times 10^{-2}$ \\
$\sigma_{1 \mathrm{ne}} / \sigma_{\text {coh }}$ & 0.012 & 0.012 \\
$\sigma_{m} / \sigma_{\text {coh }}$ & 0.21 & 0.18 \\
\hline \hline
\end{tabular}




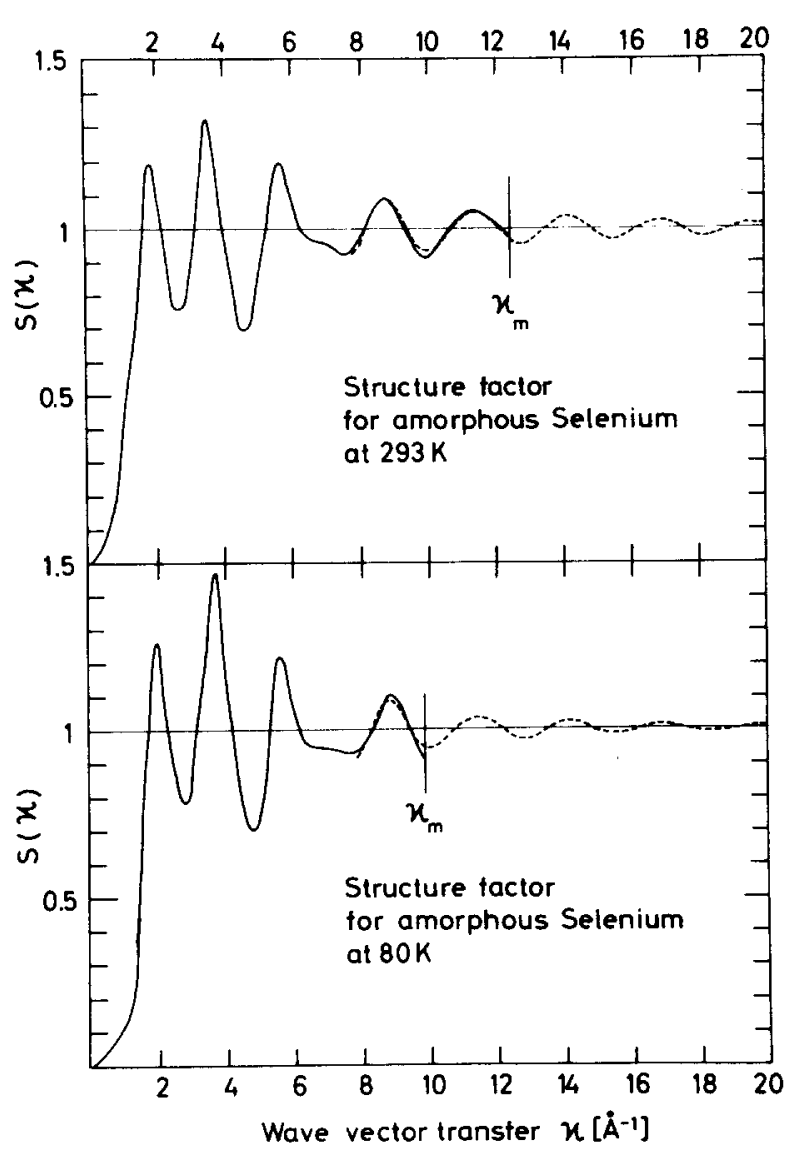

FIG. 2. Structure factor $S(\kappa)$ for amorphous selenium calculated from the intensity data as stated in the text. Dashed curve indicates the extended $S(\kappa)$ obtained through the procedure discussed in Sec. V, using the parameters listed in Table III.

Having applied this procedure, we obtained the $S(\kappa)$ function shown in Fig. 2 for amorphous selenium at the two temperatures in the region $0 \leq \kappa \leq 11 \AA^{-1}$.

\section{DETERMINATION OF $g(r)$}

We discuss now the problems involved in obtaining $g(r)$ through the Fourier transform of Eq. (1), since $S(\kappa)$ has only been measured up to a finite $\kappa_{m}$. This general truncation problem is severe because $\kappa[S(\kappa)-1]$ for selenium as it appears in Fig. 2 has not yet converged towards zero at $\kappa_{m}$. We therefore propose an extrapolation procedure for $S(\kappa)$ based on the fact that only the short distances in the sample determine $S(\kappa)$ for great $\kappa$ values. The procedure does not involve any subjective judgement of the user, in which respect the method seems superior to methods proposed by other authors.

Different methods have been used to overcome the truncation problem. To understand the nature of such errors, we suppose exact knowledge about the Fourier transform $G(r)$ of a function $F(\kappa)$, that is

$$
G(r)=\frac{2}{\pi} \int_{0}^{\infty} F(\kappa) \sin (\kappa r) d \kappa .
$$

If we do not integrate to infinity, but only to a finite value $\kappa_{m}$, it corresponds to an integration to infinity, not of the function $F(\kappa)$, but of the function $F(\kappa) D(\kappa)$, where

$$
\begin{array}{ll}
D(\kappa)=1, & \text { for } 0 \leq \kappa \leq \kappa_{m} \\
D(\kappa)=0 & \text { for } \kappa_{m}<\kappa<\infty .
\end{array}
$$

$D(\kappa)$ is called a window function. Instead of $G(r)$ we then get

$$
G^{\prime}(r)=\frac{2}{\pi} \int_{0}^{\infty} F(\kappa) D(\kappa) \sin (\kappa r) d \kappa .
$$

Using the convolution theorem we get

$$
G^{\prime}(r)=\frac{2}{\pi} \int_{0}^{\infty} Q\left(r-r^{\prime}\right) G\left(r^{\prime}\right) d r^{\prime},
$$

where $Q(r)$ is the cosine Fourier transform of $D(k)$. Equation (12) tells us that integration to $\kappa_{m}$ means that $G^{\prime}(r)$ is a weighted average of the exact $G(r)$ with $Q(r)$ as the weighting function. In the limit, where $Q(r)$ is a delta function, one gets the exact function $G(r)$ corre sponding to $\kappa_{m} \rightarrow \infty$. Since $Q(r)$ generally will have side peaks in addition to a central peak, these may introduce false peaks in $G^{\prime}(r)$, especially around the main peaks of $G(r)$. Simultaneously, fine details of $G(r)$ are smeared out according to the final width of the central peak in $Q(r)$.

Several window functions have been suggested, the aim being to minimize the two competing errors, the side peaks and the finite width of $Q(r)$.

For selenium we have tried this method by applying the Hamming window ${ }^{12}$ :

$$
\begin{aligned}
& D(\kappa)=0.54+0.46 \cos \left[\pi\left(\kappa / \kappa_{m}\right)\right], \kappa<\kappa_{m} \\
& D(\kappa)=0, \kappa \geq \kappa_{m} .
\end{aligned}
$$

The resulting $g(r)$ is shown on Fig. $3(\mathrm{~b})$. This is to be compared with Fig. 3(a), where no truncation correc-

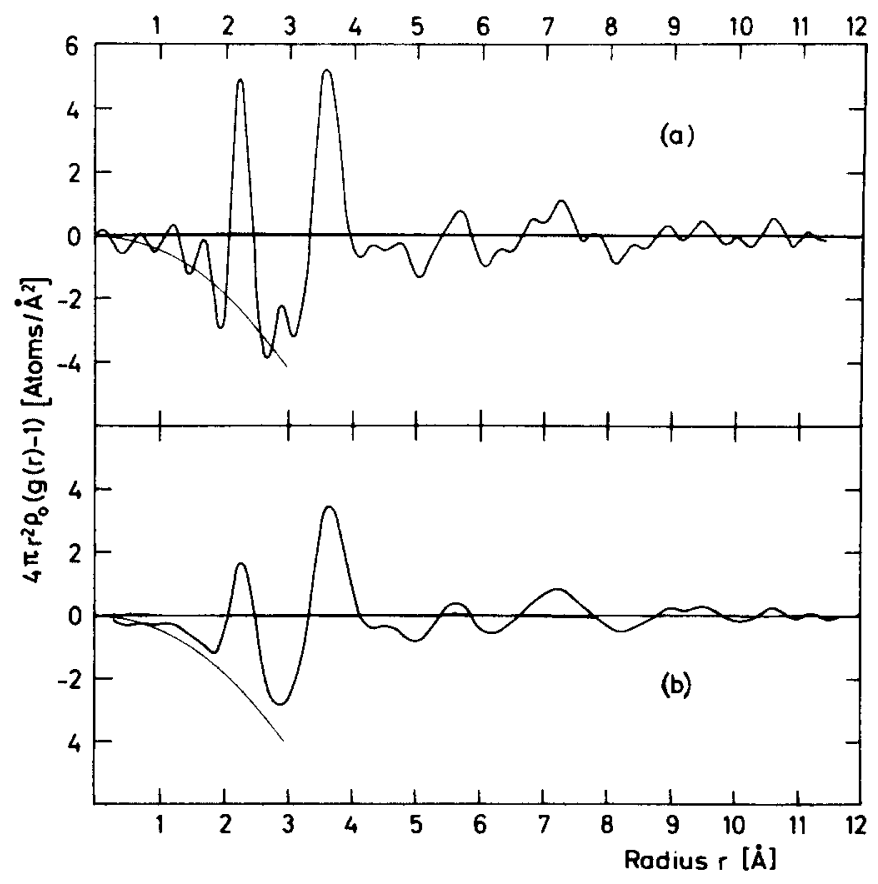

FIG. 3. $4 \pi r^{2} \rho_{0}[g(r)-1]$ for amorphous selenium at $T=293 \mathrm{~K}$, shown with unsatisfactory treatment of truncation errors. (a) No truncation correction applied. (b) The Hamming window applied to eliminate truncation errors. 
tions were applied. It is seen that the Hamming window removes symmetrically situated side peaks around the first peak of $g(r)$, but at the same time this peak is broadened considerably, implying that details of the correct $g(r)$ may be hidden by this method. These characteristics seem to be rather independent of the type of window function applied. Thus the application of this method should not be used to calculate $g(\gamma)$ quantitatively, but only as a qualitative indicator of which peaks might be false.

An alternative way, originally proposed by Kaplow et al. , ${ }^{4}$ is to make an educated guess on $S(\kappa)$ for $\kappa>\kappa_{m}$, so that the transform of Eq. (1) can be done correctly. Kaplow et al. suggested a self-consistent iterative procedure, where the idea is to study the effect of truncation of the $g(r)$ function as one truncates the structure factor data at different $\kappa$ values less than $\kappa_{m}$. From the trend in $g(r)$ one makes a guess $g^{\prime}(r)$ of the form of the final $g(r)$. This guess is then transformed to $S^{\prime}(\kappa)$, through Eq. (2), now obtained also for $\kappa>\kappa_{m}$. If the experimental $S(\kappa)$ and the calculated $S^{\prime}(\kappa)$ differ, $g^{\prime}(r)$ is modified until satisfactory accordance between $S^{\prime}(\kappa)$ and $S(\kappa)$ is obtained. Finally, one uses the calculated $S^{\prime}(\kappa)$ to give the final $g(r)$, free from truncation errors. This is not a unique mathematical procedure, as also pointed out by Kaplow. By applying this method to our data, we always obtained convergence, but the final result was dependent of the way we started the iteration.

Consequently we looked for another method to extend $S(\kappa)$ based on a better understanding of which part of $g(r)$ plays a role for large $\kappa$ 's in $S(\kappa)$. It appears from Fig. 3 that the dominant feature of $g(r)$ is the two peaks at $r_{1}$ $=2.32 \AA$ and $r_{2}=3.69 \AA$, and it may be argued from the form of Eq. (1) that $S(\kappa)$ for large $\kappa$ 's is mainly determined by $g(r)$ at these distances. Accordingly, we suppose a model for $g(r)$, where the structural element consists of two discrete Gaussian-like distributions centered around $r_{1}$ and $r_{2}$ :

$$
\begin{aligned}
4 \pi r^{2} \rho_{0} g^{m}(r)= & \frac{N_{1}}{\sqrt{2 \pi \sigma_{1}^{2}}} \frac{r}{r_{1}} \exp \frac{\left(r-r_{1}\right)^{2}}{2 \sigma_{1}^{2}} \\
& +\frac{N_{2}}{\sqrt{2 \pi \sigma_{2}^{2}}} \frac{r}{r_{2}} \exp \frac{\left(r-r_{2}\right)^{2}}{2 \sigma_{2}^{2}} .
\end{aligned}
$$

$N_{1}$ and $N_{2}$ are the numbers of nearest and next nearest neighbors to an atom, and $\sigma_{1}^{2}$ and $\sigma_{2}^{2}$ are the variances in the respective distances. The reason for not choosing a simple Gaussian distribution is purely a matter of mathematical convenience, which enables us to obtain an analytical expression for the structure factor. Outside the range of this structural element we set $g^{m}(r)$ $=1$, which again means that the upper limit of integration in Eq. (2) may be replaced by the range of the structural element. The integration in Eq. (2) may therefore be split into two parts, one which involves the integration over the structural element Eq. (13), and one over the "hole" in which the structural element is embedded. Whence

$S^{m}(\kappa)-1=N_{1} \frac{\sin \left(\kappa r_{1}\right)}{\kappa r_{1}} \exp \left(-\frac{1}{2} \sigma_{1}^{2} \kappa^{2}\right)$

$$
\begin{aligned}
& +N_{2} \frac{\sin \left(\kappa r_{2}\right)}{\kappa r_{2}} \exp \left(-\frac{1}{2} \sigma_{2}^{2} \kappa^{2}\right) \\
& +\frac{4 \pi \rho_{Q}}{\kappa^{3}}\left[\kappa r_{2} \cos \left(\kappa r_{2}\right)-\sin \left(\kappa r_{2}\right) \exp \left(-\frac{1}{2} \sigma_{2}^{2} \kappa^{2}\right),\right.
\end{aligned}
$$

where the two first terms are recognized from Eq. (13). The third term is the contribution from the hole in which the structural element was embedded. It depends on $k^{-3}$ and is negligible for the large $\kappa$ 's. The factor $\exp \left(-\frac{1}{2} \sigma_{2}^{2} \kappa^{2}\right)$ in the last term was included to ensure that $g^{m}(r)$ goes smoothly to unity outside the range of the structural element. As the contribution from such a term dies out in a similar way as the contribution from the hole, it is not important for our purpose.

Values for $N_{1}, N_{2}, \sigma_{1}$, and $\sigma_{2}$ were then found through a least square fit between our model and the measured data. In order to avoid influence of truncation effects on the comparison, one must fit the Fourier transform of $S^{m}(\kappa)$ to that of the measured $S(\kappa)$, both truncated at $\kappa_{m}$. The reliability of this treatment is illustrated in Fig. 4, where we show the resulting Fourier transforms. The feature of importance for the fit is the first peak and the left part of the second peak, which seems to be well separated from other features. The fit proved to be sensitive to the values of the parameters of the model and in this way we obtained the values as shown in Table III. The dashed curves on Fig. 2 show how smoothly $S^{m}(\kappa)$ extends the measured curve. According to $S^{m}(\kappa)$ the structure factor would show significant structure up to $\kappa=30 \AA^{-1}$. Using our measured data for $S(\kappa)$ for $\kappa<\kappa_{m}$ and our model for $\kappa>\kappa_{m}$, we obtain through Eq. (1) the pair distribution function $g(r)$ for amorphous selenium at 80 and $293 \mathrm{~K}$. The two functions are shown in Fig. 5 . For comparison, we also show schematically in Fig. 5 the number of neighboring atoms for the three crystalline phases of selenium.

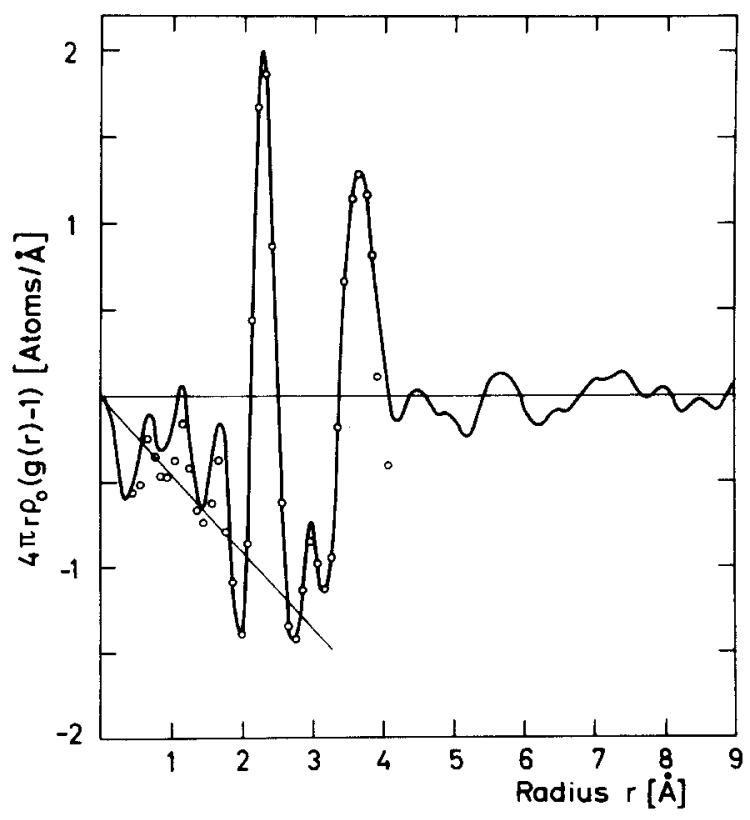

FIG. 4. $4 \pi r \rho_{0}[g(r)-1]$ shown for direct Fourier transform of the measured $S(\kappa)$ (solid line), and for proposed model (open circles). 
TABLE III. Final data used for extension of the structure factors.

\begin{tabular}{ccccccc}
\hline \hline$T[\mathrm{~K}]$ & $r_{1}[\AA]$ & $r_{2}[\AA]$ & $\sigma_{1}[\AA]$ & $\sigma_{2}[\AA]$ & $N_{1}$ & $N_{2}$ \\
\hline 293 & 2.32 & 3.69 & 0.068 & 0.257 & 1.95 & 6.9 \\
80 & 2.32 & 3.69 & 0.095 & 0.235 & 1.95 & 7.3
\end{tabular}

\section{DISCUSSION}

As a result of our measurements we get the structure factor $S(\kappa)$ for amorphous selenium at room temperature and at liquid nitrogen temperatures, as well as the two neutron scattering cross sections $\sigma_{\mathrm{con}}$ and $\sigma_{\mathrm{inc}} . S(\kappa)$ is shown in Fig. 2, and tabulated in Table V. Concerning the accuracy of the measured $S(\kappa)$, the statistical rms uncertainty is $\sim 0.5 \%$ after smoothing. The absorption and the Placzek corrections are both well determined, and we estimate them to contribute the uncertainty of $S(\kappa)$, each within $0.5 \%$. Although our treatment of the multiple scattering is not fully satisfactory, the applied incoherent approximation should hold relatively well because $S(\kappa)$ does not deviate much from unity. The magnitude of the incoherent cross section $\sigma_{1 \mathrm{nc}}$ which enters Eq. (4) in the same way as $\sigma_{m}$ indicates that $\left(\sigma_{m}+\sigma_{1 \mathrm{ic}}\right) /$ $\sigma_{\text {coh }}$ is accurate within $1 \%$. We therefore estimate $\sigma_{\text {coh }}$ and the structure factors to be determined within $\pm 1.5 \%$.

Considering the structure factor, when the temperature is lowered from $293 \mathrm{~K}$, which is only $30 \mathrm{~K}$ below the glass-transition temperature, to $80 \mathrm{~K}$, close to the Debye temperature of $75 \mathrm{~K}$, only small qualitative changes occur. The only feature of importance, in agreement with Kaplow et al., seems to be a slight sharpening of the two first peaks in $S(\kappa)$ when the temperature is lowered. The consequent changes in the structure will be discussed below.

The obtained values of the cross sections in Sec. III fall within previously reported values, and we find this method interesting since it involves no absolute scattering measurement, but rather the use of analytical properties of $g(r)$. As mentioned above, $\sigma_{1 \mathrm{nc}}$ appears in Eq. (4) similarly to $\sigma_{m}$, which is dominant. The value of $\sigma_{\text {inc }}$ is consequently rather poorly determined, but the fact that it is small serves as a consistency check on the applied method.

The obtained $g(r)$ 's, shown in Fig. 5 (see Table VI) are characterized by position and shape of the two first peaks and a number of peak positions, shown in Table IV. The natural widths of the two first peaks have to be compared with the resolution broadening discussed in Sec. IV owing to the finite $\kappa_{m}$. By our method we have corrected for this instrumental effect, which may be described as an effective instrumental width $\sigma_{\text {ins }}=0.51 / \kappa_{m}$, i.e., 0.041 and 0.054 for 293 and $80 \mathrm{~K}$, respectively. In both cases the measured natural width of the first peak is more than 50\% larger than the resolution.

However, the residual wiggles in $g(r)$ after the corrections show that still some uncertainty is left.

Mountain ${ }^{13}$ has pointed out that one cannot in an easy way relate the uncertainties in $S(\kappa)$ and $g(r)$, but $g(r)$ is extremely sensitive to systematic errors in $S(\kappa)$. In this respect the work of Kaplow et al. and our work seem satis factory, and it is therefore of interest to compare the details of the pair-distribution functions. Kaplow et al. used $\mathrm{x}$-ray measurements and a different method of extending the structure factor data to eliminate truncation errors. From Table III it is seen that the numbers of nearest and next nearest neighbors a re the same for both cold and warm. selenium. It is also seen that the width of the first peak is increased with decreasing temperatures, which is surprising. The room temperature data agree with those of Kaplow, but he did not observe the broadening of the first peak for cold selenium. The width of the peaks is due to structural disorder as well as to thermal motion of the atoms, and one would expect the first peak es-

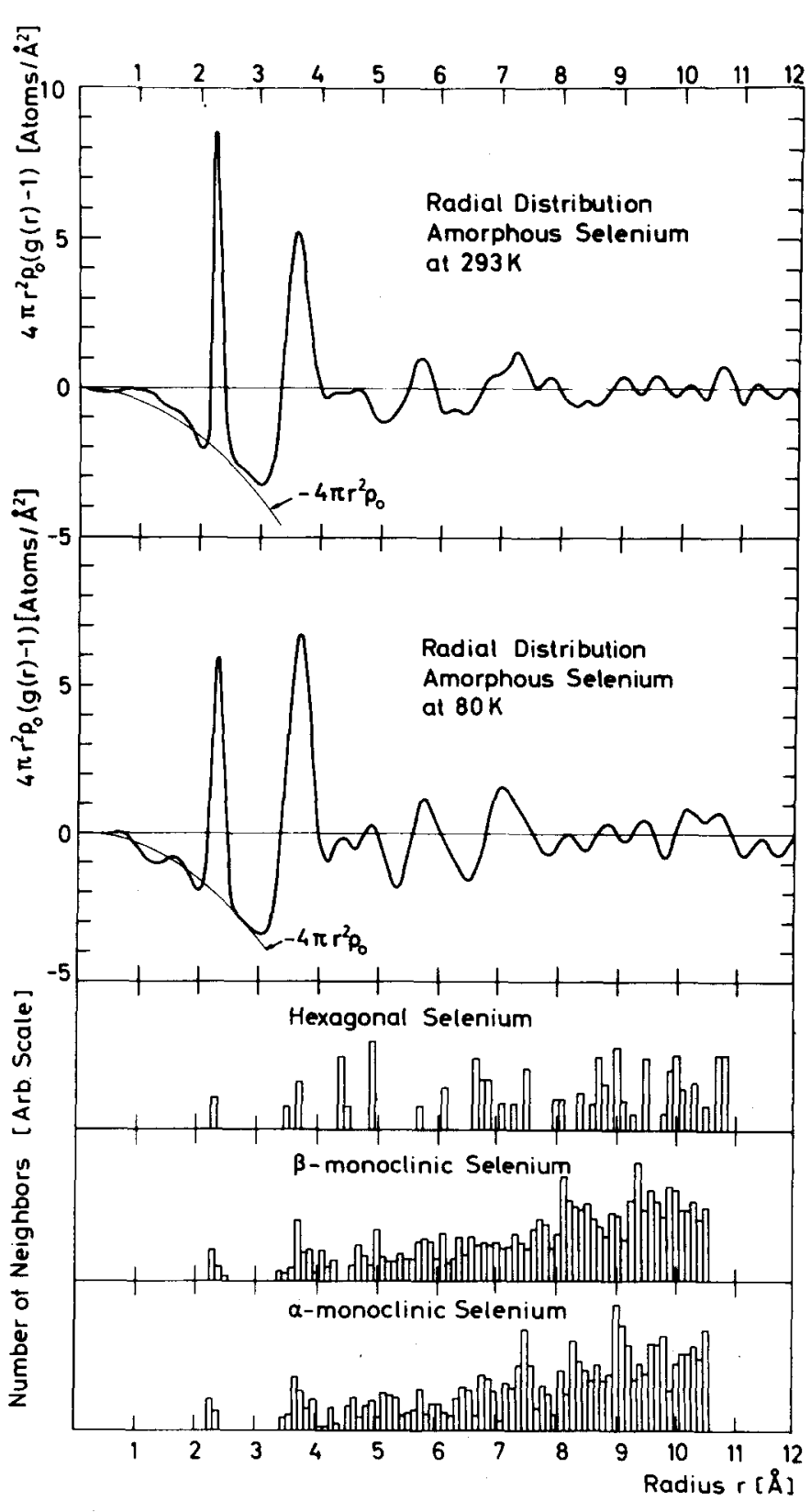

FIG. 5. $4 \pi r^{2} \rho_{0}[g(r)-1]$ for amorphous selenium at 80 and 293 K. Final result. Also shown schematically, the number of surrounding atoms in the three crystalline phases. 
TABLE IV. Peak positions in the pair-distribution functions obtained by different authors through $\mathrm{x}-\mathrm{ray}$ and neutron diff $\mathrm{raction}$ at different temperatures.

\begin{tabular}{|c|c|c|c|c|c|c|c|c|c|c|}
\hline \multicolumn{3}{|c|}{ Richter (Ref. 3) } & \multicolumn{4}{|c|}{ Henninger et al (Ref. 1) } & \multicolumn{2}{|c|}{ Kaplow ef al (Ref. 4) } & \multicolumn{2}{|c|}{ This work } \\
\hline $\begin{array}{l}\text { Se(I) } \\
\text { liq. air temp. } \\
\lambda=1.54[\AA] \\
\kappa_{m}=7.9\left[\AA^{-1}\right]\end{array}$ & $\begin{array}{l}\text { Se (II) } \\
T=293 \mathrm{~K} \\
\lambda=1.54[\hat{\mathrm{A}}] \\
\kappa_{m}=7.9\left[\mathrm{~A}^{-1}\right]\end{array}$ & $\begin{array}{l}\text { Se (IHI) } \\
T=293 \mathrm{~K} \\
\lambda=0.56[\mathrm{~A}] \\
k_{m}=7.9\left[\mathrm{~A}^{-1}\right]\end{array}$ & $\begin{array}{l}\text { x-ray } \\
T=293 \mathrm{~K} \\
\lambda=0.56[\AA] \\
\kappa_{m}=13\left[\AA^{-1}\right]\end{array}$ & $\begin{array}{l}\text { x-ray } \\
T=293 \mathrm{~K} \\
\lambda=0.56[\hat{A}] \\
\kappa_{m}=13\left[\hat{A}^{-1}\right]\end{array}$ & $\begin{array}{l}\text { Neutron } \\
T=293 \mathrm{~K} \\
\lambda=1.083[\AA] \\
\kappa_{m}=13\left[\hat{A}^{-1}\right]\end{array}$ & $\begin{array}{l}\text { Neutron } \\
T=293 \mathrm{~K} \\
\lambda=0.797[\AA] \\
\kappa_{m}=13\left[\mathrm{~S}^{-1}\right]\end{array}$ & $\begin{array}{l}\text { x-ray } \\
T=293 \mathrm{~K} \\
\lambda=1.792[\AA] \\
\lambda=0.61[\AA] \\
k_{m}=15\left[\AA^{-1}\right]\end{array}$ & $\begin{array}{l}x-\text { ray } \\
T=79 \mathrm{~K} \\
\lambda=1.792[\mathrm{~K}] \\
\lambda=0.61[\mathrm{~A}] \\
\kappa_{m}=15\left[\mathrm{~A}^{-1}\right]\end{array}$ & $\begin{array}{l}\text { Neutron } \\
T=293 \mathrm{~K} \\
\lambda=1.705[\AA] \\
\lambda=0.852[\AA] \\
\kappa_{m}=125\left[\AA^{-1}\right]\end{array}$ & $\begin{array}{l}\text { Neutron } \\
T=80 \mathrm{~K} \\
\lambda=1.7116[\mathrm{~A}] \\
\lambda=09563[\mathrm{~A}] \\
k_{m}=9.9\left[\mathrm{~A}^{-1}\right]\end{array}$ \\
\hline $\begin{array}{c}2.35 \\
- \\
3.68\end{array}$ & $\begin{array}{c}2.35 \\
- \\
3.78\end{array}$ & $\begin{array}{l}2.35 \\
3.0 \\
3.55\end{array}$ & $\begin{array}{c}2.33 \\
- \\
3.73\end{array}$ & $\begin{array}{c}2.33 \\
- \\
3.73\end{array}$ & $\begin{array}{c}2.31 \\
- \\
3.72\end{array}$ & $\begin{array}{l}2.33 \\
3.0 \\
3.71\end{array}$ & $\begin{array}{c}2.34 \\
- \\
3.75\end{array}$ & $\begin{array}{c}2.34 \\
- \\
3.75\end{array}$ & $\begin{array}{c}2.32 \\
- \\
3.69\end{array}$ & $\begin{array}{c}2.32 \\
- \\
3.69\end{array}$ \\
\hline $\begin{array}{l}4.3 \\
4.7 \\
5.25 \\
5.75 \\
6.2\end{array}$ & $\begin{array}{l}- \\
4.8 \\
- \\
5.75 \\
6.5\end{array}$ & $\begin{array}{c}4.5 \\
5.0 \\
- \\
5.6 \\
6.1\end{array}$ & $\begin{array}{c}4.65 \\
- \\
- \\
5.80 \\
-\end{array}$ & $\begin{array}{c}4.62 \\
- \\
- \\
5.8 \\
-\end{array}$ & $\begin{array}{c}4.58 \\
- \\
- \\
5.9 \\
-\end{array}$ & $\begin{array}{l}4.5 \\
5.0 \\
- \\
5.75 \\
6.3\end{array}$ & $\begin{array}{l}4.3 \\
4.7 \\
5.1 \\
5.8 \\
6.4\end{array}$ & $\begin{array}{l}4.3 \\
4.7 \\
5.1 \\
5.8 \\
-\end{array}$ & $\begin{array}{l}4.3 \\
4.7 \\
- \\
5.75 \\
6.3\end{array}$ & $\begin{array}{l}4.4 \\
4.9 \\
- \\
5.75 \\
-\end{array}$ \\
\hline $\begin{array}{c}7.1 \\
- \\
-\end{array}$ & $\begin{array}{c}7.4 \\
- \\
-\end{array}$ & $\begin{array}{c}7.0 \\
- \\
-\end{array}$ & $\begin{array}{l}6.7 \\
7.3 \\
7.9\end{array}$ & $\begin{array}{l}7.0 \\
7.4 \\
7.9\end{array}$ & $\begin{array}{l}6.8 \\
7.2 \\
7.9\end{array}$ & $\begin{array}{l}6.9 \\
7.4 \\
8.1\end{array}$ & $\begin{array}{l}7.0 \\
7.5 \\
7.9\end{array}$ & $\begin{array}{c}- \\
7.3 \\
-\end{array}$ & $\begin{array}{l}6.8 \\
7.35 \\
7.9\end{array}$ & $\begin{array}{c}- \\
7.1 \\
-\end{array}$ \\
\hline 8.2 & - & - & - & - & - & - & $\begin{array}{l}8.5 \\
9.2\end{array}$ & $\begin{array}{l}8.5 \\
9.2\end{array}$ & $\begin{array}{l}8.4 \\
9.0\end{array}$ & $\begin{array}{l}8.2 \\
8.9\end{array}$ \\
\hline $\begin{array}{l}9.1 \\
-\end{array}$ & $\begin{array}{l}9.2 \\
-\end{array}$ & - & - & - & - & - & 9.6 & - & 9.5 & 9.4 \\
\hline - & - & - & - & - & - & - & - & 10.1 & 10.1 & 10.1 \\
\hline - & - & - & - & - & - & - & 10.6 & 10.5 & 10.7 & 10.8 \\
\hline
\end{tabular}

pecially to be narrow on cooling since it mainly corresponds to a covalent bond. This simple dynamical interpretation does not seem to be adequate since we observe the opposite effect. We must conclude that both structural and dynamical disorder contributes to the width of the first peak in $g(r)$. Since the major changes in $S(\kappa)$ on cooling seem to be the same in both measurements, the difference may be because of defects in the data treatment. Further, our measurements were performed with the same sample at both temperatures, whereas Kaplow used two samples; the one at low temperature was vapor-deposited, and this may have introduced some surface effects which can also be responsible for the discrepancy.

The difference between our results and those of Ref. 1 at room temperature may be a result of differences in the correction procedures of the intensity data (absorption-correction) and to differences in the treatment of the truncation errors. Thus, they found that the first peak in the $4 \pi \rho_{0} r^{2}[g(r)-1]$ curve was lower than the second peak at room temperature, a result we would get if we completely neglected truncation errors [Fig. 3(a)].

The $\mathrm{x}$-ray results of $\mathrm{Richter}^{3}$ differ in two important ways from our results. Firstly, the second peak in the $4 \pi \rho_{0} r^{2}[g(r)-1]$ curve is much higher than the first peak, and the peaks are broader than we found them. This is probably because of large truncation errors, as $\kappa_{m}$ in these measurements is only $7.9 \AA^{-1}$. Secondly, Richter distinguishes between three different types of selenium, corresponding to three slightly different positions of the second peak in the pair-distribution function (see Table IV). To our knowledge no other authors have observed these three different forms of selenium.

From the above discussions we have seen that it is important to know the experimental $\kappa_{m}$ values when one compares different pair-distribution functions, which have not been corrected for truncation errors. If one compares the distribution functions obtained from dif- ferent measurements with different $\kappa_{m}$ values, it is valuable to have a general procedure, e.g., the one suggested here, to make the functions comparable. Otherwise one may easily draw conclusions which are not physically based.

Besides the two first peaks in $g(r)$, structure persists up to the largest distances shown in Fig. 5. Further, it is seen from Fig. 3 that in the region $4 \AA<r<11 \AA$ the gross features of $g(r)$ are relatively independent of the way in which truncation errors are treated. This indicates the significance of the peak positions in the measured $g(r)$. By comparison to other published results it is therefore instructive to notice the agreement between the peak positions also for larger distances. In Table IV are shown the peak positions reported from earlier diffraction experiments on amorphous selenium both at 298 and $80 \mathrm{~K}$. It seems to us that if one uses either our method or the one proposed by Kaplow, one can resolve peaks as far as $11 \AA$ from experiment. This immediately calls for more sophisticated ways of obtaining models, both of the type based on a random generation or based on hand built models, since none of those has so far been able to produce reliable data for so large distances.

Finally, we will make a short discussion of the atomic arrangements in amorphous selenium. Recently, Richte $\mathrm{r}^{3}$ suggested a model for amorphous selenium, which is in accordance with the distances found in their pairdistribution function. It is suggested that amorphous selenium consists of planar zigzag chains joined to lay ers in the distance of approximately 3.69 $\AA$. The structural element of the chains is the same isosceles triangle as is found for the screw chain in hexagonal crystalline selenium.

One may now ask if this model is reasonable also from an energetic point of view. The heat of crystallization of amorphous selenium has been determined by differential thermal analysis to be $\sim 1 \mathrm{kcal} / \mathrm{g}$ atom. ${ }^{14}$ To make 
TABLE V. Values of the structure factor $\mathrm{S}(\kappa)$ for selenium at the temperatures $T=293$ and $80 \mathrm{~K}$, as a function of the wave vector transfer.

\begin{tabular}{|c|c|c|c|c|c|c|c|c|}
\hline$\kappa \AA^{-1}$ & $\begin{array}{l}S(\kappa) \\
(T=293 \mathrm{~K})\end{array}$ & $\begin{array}{l}S(\kappa) \\
(T=80 \mathrm{~K})\end{array}$ & $\kappa \AA^{-1}$ & $\begin{array}{l}S(\kappa) \\
(T=293 \mathrm{~K})\end{array}$ & $\begin{array}{l}S(\kappa) \\
(T=80 \mathrm{~K}) \\
\end{array}$ & $\kappa \AA^{-1}$ & $\begin{array}{l}S(K) \\
(T=293 \mathrm{~K})\end{array}$ & $\begin{array}{l}S(\kappa) \\
(T=80 \mathrm{~K})\end{array}$ \\
\hline 0.05 & 0.009 & 0.009 & 4.25 & 0.870 & 0.957 & 8.45 & 1.041 & 1.028 \\
\hline 0.15 & 0.010 & 0.015 & 4.35 & 0.802 & 0.881 & 8.55 & 1.055 & 1.045 \\
\hline 0.25 & 0.015 & 0.030 & 4.45 & 0.736 & 0.824 & 8.65 & 1.072 & 1.071 \\
\hline 0.35 & 0.030 & 0.041 & 4.55 & 0.709 & 0.771 & 8.75 & 1.096 & 1.090 \\
\hline 0.45 & 0.040 & 0.046 & 4.65 & 0.695 & 0.731 & 8.85 & 1.100 & 1.113 \\
\hline 0.55 & 0.070 & 0.060 & 4.75 & 0.705 & 0.700 & 8.95 & 1.091 & 1.104 \\
\hline 0.65 & 0.100 & 0.070 & 4.85 & 0.710 & 0.705 & 9.05 & 1.096 & 1.091 \\
\hline 0.75 & 0.160 & 0.081 & 4.95 & 0.744 & 0.731 & 9.15 & 1.071 & 1.082 \\
\hline 0.85 & 0.230 & 0.094 & 5.05 & 0.796 & 0.779 & 9.25 & 1.055 & 1.064 \\
\hline 0.95 & 0.310 & 0.111 & 5.15 & 0.874 & 0.868 & 9.35 & 1.027 & 1.038 \\
\hline 1.05 & 0.360 & 0.131 & 5.25 & 0.928 & 0.951 & 9.45 & 1.004 & 1.012 \\
\hline 1.15 & 0.411 & 0.167 & 5.35 & 1.021 & 1.061 & 9.55 & 0.990 & 0.990 \\
\hline 1.25 & 0.483 & 0.251 & 5.45 & 1.088 & 1.158 & 9.65 & 0.946 & 0.960 \\
\hline 1.35 & 0.609 & 0.411 & 5.55 & 1.145 & 1.210 & 9.75 & 0.944 & 0.930 \\
\hline 1.45 & 0.695 & 0.579 & 5.65 & 1.181 & 1.219 & 9.85 & 0.928 & 0.912 \\
\hline 1.55 & 0.826 & 0.710 & 5.75 & 1.196 & 1.197 & 9.95 & 0.917 & \\
\hline 1.65 & 0.938 & 0.854 & 5.85 & 1.160 & 1.144 & 10.05 & 0.924 & \\
\hline 1.75 & 1.053 & 0.981 & 5.95 & 1.156 & 1.095 & 10.15 & 0.913 & \\
\hline 1.85 & 1.161 & 1.132 & 6.05 & 1.119 & 1.060 & 10.25 & 0.929 & \\
\hline 1.95 & 1.192 & 1.260 & 6.15 & 1.067 & 1.011 & 10.35 & 0.941 & \\
\hline 2.05 & 1.168 & 1.247 & 6.25 & 1.033 & 0.989 & 10.45 & 0.941 & \\
\hline 2.15 & 1.082 & 1.135 & 6.35 & 1.003 & 0.967 & 10.55 & 0.963 & \\
\hline 2.25 & 0.974 & 1.042 & 6.45 & 0.981 & 0.958 & 10.65 & 0.995 & \\
\hline 2.35 & 0.892 & 0.957 & 6.55 & 0.977 & 0.949 & 10.75 & 0.993 & \\
\hline 2.45 & 0.827 & 0.899 & 6.65 & 0.968 & 0.947 & 10.85 & 0.991 & \\
\hline 2.55 & 0.789 & 0.851 & 6.75 & 0.964 & 0.945 & 10.95 & 1.030 & \\
\hline 2.65 & 0.768 & 0.820 & 6.85 & 0.945 & 0.945 & 11.05 & 1.024 & \\
\hline 2.75 & 0.762 & 0.789 & 6.95 & 0.972 & 0.944 & 11.15 & 1.051 & \\
\hline 2.85 & 0.769 & 0.789 & 7.05 & 0.957 & 0.944 & 11.25 & 1.053 & \\
\hline 2.95 & 0.797 & 0.851 & 7.15 & 0.974 & 0.943 & 11.35 & 1.061 & \\
\hline 3.05 & 0.847 & 0.913 & 7.25 & 0.941 & 0.943 & 11.45 & 1.053 & \\
\hline 3.15 & 0.903 & 0.997 & 7.35 & 0.943 & 0.939 & 11.55 & 1.041 & \\
\hline 3.25 & 1.013 & 1.072 & 7.45 & 0.934 & 0.939 & 11.65 & 1.047 & \\
\hline 3.35 & 1.136 & 1.192 & 7.55 & 0.920 & 0.935 & 11.75 & 1.037 & \\
\hline 3.45 & 1.239 & 1.320 & 7.65 & 0.927 & $0.930^{\circ}$ & 11.85 & 1.039 & \\
\hline 3.55 & 1.312 & 1.399 & 7.75 & 0.928 & 0.930 & 11.95 & 1.027 & \\
\hline 3.65 & 1.323 & 1.435 & 7.85 & 0.938 & 0.938 & 12.05 & 1.029 & \\
\hline 3.75 & 1.284 & 1.470 & 7.95 & 0.957 & 0.947 & 12.15 & 1.010 & \\
\hline 3.85 & 1.217 & 1.364 & 8.05 & 0.968 & 0.960 & 12.25 & 1.000 & \\
\hline 3.95 & 1. 108 & 1.235 & 8.15 & 0.964 & 0.972 & 12.35 & 0.992 & \\
\hline 4.05 & 1.026 & 1.116 & 8.25 & 0.998 & 0.985 & 12.45 & 0.987 & \\
\hline 4.15 & 0.938 & 1.036 & 8.35 & 1.023 & 1.007 & & & \\
\hline
\end{tabular}

the hexagonal screw chain planar, one has to change the dihedral angle in the chain. The barrier for this rotation is estimated ${ }^{15}$ to be of the order $10 \mathrm{kcal} / \mathrm{g}$ atom to be compared with the above measured value. We therefore suggest that at least the whole sample cannot be built by planar chains, although parts of the sample may contain planar chains.

Kaplow et al. ${ }^{4}$ have made a Monte Carlo calculation of the structure of amorphous selenium making a fit to the experimental $g(r)$. They considered a model based on modifications of the crystalline phases, schematically characterized in Fig. 5, and from energetic arguments they selected the ones which showed a minimum spread in the angle between an atom and its nearest neighbors. This spread corresponds closely to the $\sigma_{2}$ in our model. They did not take the dihedral angle into account, and as the energy associated with a rotation in this angle is rather high, it may change their results, when included in the calculations. They concluded that the structure consisted mainly of slightly distorted $\mathrm{Se}_{8}$ rings, along with an occasional ring which is opened sufficiently to develop a weak localized trigonal symmetry or a few greatly deformed chains. Although they are able to reproduce the measured $g(r)$ for smaller distances, further refinements may be obtained considering the significance of the larger distances, which is indicated by comparison with our results.

\section{CONCLUSION}

Accurate structure factors and pair-distribution functions for amorphous selenium at 293 and $80 \mathrm{~K}$ have been obtained using neutron diffraction. (See Tables $\mathrm{V}$ and VI.) The systematic errors have been treated carefully and a new method to avoid truncation errors has been 
TABLE VI. Values of $f=4 \pi r^{2} \rho_{0}[g(r)-1]$ for amorphous selenium at the temperatures $T=293$ and $80 \mathrm{~K}$, as function of the distance $r$.

\begin{tabular}{|c|c|c|c|c|c|c|c|c|}
\hline$r \AA^{-1}$ & $\begin{array}{l}f \\
(T=293 \mathrm{~K})\end{array}$ & $\begin{array}{l}f \\
(T=80 \mathrm{~K})\end{array}$ & $r \AA^{-1}$ & $\begin{array}{l}f \\
(T=293 \mathrm{~K})\end{array}$ & $\begin{array}{l}f \\
(T=80 \mathrm{~K})\end{array}$ & $r \AA^{-1}$ & $\begin{array}{l}f \\
(T=293 \mathrm{~K})\end{array}$ & $\begin{array}{l}f \\
(T=80 \mathrm{~K})\end{array}$ \\
\hline 0.05 & -0.005 & -0.058 & 5.05 & -1.119 & -0.190 & 10.05 & -0.085 & 0.415 \\
\hline 0.15 & -0.042 & -0.141 & 5.15 & -1.075 & -0.991 & 10.15 & 0.084 & 0.811 \\
\hline 0.25 & -0.107 & -0.048 & 5.25 & -0.901 & -1.679 & 10.25 & 0.016 & 0.803 \\
\hline 0.35 & -0.171 & -0.059 & 5.35 & -0.645 & -1.760 & 10.35 & -0.258 & 0.604 \\
\hline 0.45 & -0.198 & -0.049 & 5.45 & -0.311 & -1.146 & 10.45 & -0.370 & 0.469 \\
\hline 0.55 & -0.184 & -0.014 & 5.55 & 0.261 & -0.112 & 10.55 & 0.023 & 0.580 \\
\hline 0.65 & -0.166 & 0.008 & 5.65 & 0.858 & 0.776 & 10.65 & 0.591 & 0.762 \\
\hline 0.75 & -0.167 & -0.019 & 5.75 & 0.987 & 1.178 & 10.75 & 0.799 & 0.765 \\
\hline 0.85 & -0.171 & -0.146 & 5.85 & 0.548 & 1.001 & 10.85 & 0.456 & 0.398 \\
\hline 0.95 & -0.150 & -0.374 & 5.95 & -0.214 & 0.537 & 10.95 & -0.190 & -0.182 \\
\hline 1.05 & -0.106 & -0.663 & 6.05 & -0.798 & 0.013 & 11.05 & -0.511 & -0.668 \\
\hline 1.15 & -0.132 & -0.925 & 6.15 & -0.873 & -0.401 & 11.15 & -0.293 & -0.782 \\
\hline 1.25 & -0.283 & -1.062 & 6.25 & -0.747 & -0.803 & 11.25 & 0.058 & -0.554 \\
\hline 1.35 & -0.477 & -1.049 & 6.35 & -0.740 & -1.197 & 11.35 & 0.182 & -0.248 \\
\hline 1.45 & -0.630 & -0.918 & 6.45 & -0.833 & -1.537 & 11.45 & -0.030 & -0.155 \\
\hline 1.55 & -0.691 & -0.825 & 6.55 & -0.840 & -1.570 & 11.55 & -0.315 & -0.343 \\
\hline 1.65 & -0.741 & -0.852 & 6.65 & -0.503 & -1.161 & 11.65 & -0.317 & -0.608 \\
\hline 1.75 & -0.995 & -1.110 & 6.75 & 0.013 & -0.340 & 11.75 & -0.169 & -0.698 \\
\hline 1.85 & -1.396 & -1.452 & 6.85 & 0.308 & 0.587 & 11.85 & -0.104 & -0.493 \\
\hline 1.95 & -1.759 & -1.835 & 6.95 & 0.387 & 1. 288 & 11.95 & -0.167 & -0.176 \\
\hline 2.05 & -2.045 & -1.889 & 7.05 & 0.440 & 1.564 & 12.05 & -0.314 & 0.025 \\
\hline 2.15 & -1.638 & -0.594 & 7.15 & 0.661 & 1.463 & 12.15 & -0.326 & -0.058 \\
\hline 2.25 & 4.520 & 4.004 & 7.25 & 1.024 & 1.181 & 12,25 & -0.192 & -0.275 \\
\hline 2.35 & 8.363 & 5.765 & 7.35 & 1.109 & 0.855 & 12.35 & -0.105 & -0.415 \\
\hline 2.45 & -0.448 & 1.135 & 7.45 & 0.780 & 0.525 & 12.45 & -0.027 & -0.274 \\
\hline 2.55 & -3.389 & -2.054 & 7.55 & 0.325 & 0.119 & 12.55 & 0.056 & 0.039 \\
\hline 2.65 & -2.600 & -2.749 & 7.65 & 0.041 & -0.312 & 12.65 & 0.125 & 0.334 \\
\hline 2.75 & -2.774 & -3.006 & 7.75 & 0.123 & -0.665 & 12.75 & 0.190 & 0.374 \\
\hline 2.85 & -2.873 & -3.167 & 7.85 & 0.334 & -0.735 & 12.85 & 0.092 & 0.205 \\
\hline 2.95 & -3.124 & -3.359 & 7.95 & 0.278 & -0.533 & 12.95 & -0.097 & -0.016 \\
\hline 3.05 & -3.293 & -3.455 & 8.05 & -0.018 & -0.188 & 13.05 & -0.199 & -0.065 \\
\hline 3.15 & -3.115 & -3.304 & 8.15 & -0.369 & -0.011 & 13.15 & -0.248 & 0.061 \\
\hline 3.25 & -2.412 & -2.440 & 8.25 & -0.562 & -0.100 & 13.25 & -0.230 & 0.248 \\
\hline 3.35 & -0.773 & -0.635 & 8.35 & -0.500 & -0.394 & 13.35 & -0.128 & 0.323 \\
\hline 3.45 & 1,608 & 2.079 & 8.45 & -0.441 & -0.577 & 13.45 & 0.075 & 0.278 \\
\hline 3.55 & 3.880 & 4.807 & 8.55 & -0.501 & -0.473 & 13.55 & 0.483 & 0.230 \\
\hline 3.65 & 5.140 & 6.423 & 8.65 & -0.551 & -0.086 & 13.65 & 0.839 & 0.305 \\
\hline 3.75 & 4.716 & 6.014 & 8.75 & -0.485 & 0.283 & 13.75 & 0.768 & 0.488 \\
\hline 3.85 & 2.986 & 3.948 & 8.85 & -0.154 & 0.389 & 13.85 & 0.277 & 0.590 \\
\hline 3.95 & 1.148 & 1.374 & 8.95 & 0.292 & 0.170 & 13.95 & -0.353 & 0.455 \\
\hline 4.05 & -0.004 & -0.363 & 9.05 & 0.489 & -0.128 & 14.05 & -0.633 & 0.071 \\
\hline 4.15 & -0.300 & -0.900 & 9.15 & 0.375 & -0.213 & 14.15 & -0.374 & -0.320 \\
\hline 4.25 & -0.169 & -0.611 & 9.25 & 0.069 & 0.019 & 14.25 & 0.004 & -0.491 \\
\hline 4.35 & -0.157 & -0.276 & 9.35 & -0.128 & 0.369 & 14.35 & 0.158 & -0.337 \\
\hline 4.45 & -0.197 & -0.228 & 9.45 & 0.054 & 0.487 & 14.45 & 0.069 & -0.075 \\
\hline 4.55 & -0.133 & -0.398 & 9.55 & 0.0375 & 0.206 & 14.55 & -0.065 & 0.051 \\
\hline 4.65 & -0.066 & -0.420 & 9.65 & 0.492 & -0.342 & 14.65 & 0.029 & -0.121 \\
\hline 4.75 & -0.115 & -0.163 & 9.75 & 0.291 & -0.742 & 14.75 & 0.183 & -0.395 \\
\hline 4.85 & -0.455 & 0.194 & 9.85 & -0.103 & -0.707 & 14.85 & 0.122 & -0.500 \\
\hline 4.95 & -0.918 & 0.263 & 9.95 & -0.265 & -0.203 & 14.95 & -0.036 & -0.214 \\
\hline
\end{tabular}

introduced. Good agreement with earlier measurements on the peak positions for distances less than $11 \AA$ is obtained, but some finer details as the width of the first peak at small temperatures disagree.

The experimental data have been used to find values for the coherent and the incoherent scattering cross section.

\section{ACKNOWLEDGMENTS}

We wish to thank Professor J. W. Wlkins, Cornell University, who initiated this study, and Professor
R. O. Pohl, Cornell University, for providing the samples and for a valuable discussion. Further, we are grateful to Bente Lebech and Mourits Nielsen for discussions and criticism of the manuscript.

†Present address: Physics Department, Brookhaven National Laboratory, Upton, L. I. , N. Y., 11973. On leave from Physics Laboratory I, University of Copenhagen, Denmark. ${ }^{1}$ E. H. Henninger, R. C. Buschart, and L. Heaton, J. Chem. Phys. 46, 586 (1967). 
${ }^{2}$ J. Moscinski, A. Renninger, and B. L. Averbach, Phys. Lett. A 42, 453 (1973).

${ }^{3}$ H. Richter, J. Non-Cryst. Solids 8, 338 (1972).

${ }^{4}$ R. Kaplow, T. A. Rowe, and B. L. Averbach, Phys. Rev. 168, 1068 (1968).

${ }^{5}$ A. L. Renninger and B. L. Averbach, Phys. Rev. B 3, 1507 (1974).

${ }^{6}$ L. A. de Graaf and B. Mozer, J. Chem. Phys, 55, 4967 (1971).

${ }^{7}$ International Tables for X-ray Crystallography; (Kynoch, Birmingham, England, 1972), p. 291.

${ }^{8}$ I. A. Blech and B. L. Averbach, Phys. Rev. 137, A1113 (1965).

${ }^{9}$ G. Placzek, Phys. Rev. 86, 377 (1952); J. L. Yarnell, M. J.
Katz, R. G. Wenzel, and S. H. Koenig, Phys. Rev. A 7, 2130 (1973).

${ }^{10}$ G. E. Bacon, Neutron Diffraction (Oxford University, London, 1958), p. 102; see also a report by P. A. Egelstaff (AERE N/R 1147, Harwell, 1958).

${ }^{11}$ R. Kaplow, S. L. Strong, and B. L. Averbach, Acta Crystallogr. 19, 1043 (1965).

${ }^{12}$ R. B. Blackmann and J. W. Tukey, The Measurement of Power Spectra (Dover, New York, 1958), p. 14.

${ }^{13}$ R. D. Mountain, J. Chem. Phys. 57, 4346 (1972).

${ }^{14} \mathrm{~B}$. Dobovisek, L. A. Mechkovskii, A. Rosina, Chem. Abst. 69, 110842 (p. 10389, 1968).

${ }^{15}$ P. J. Flory, Statistical Mechanic of Chain Molecules (Interscience, New York, 1969), p. 157 and references therein. 